\title{
Launch Vehicle Control Center Architectures
}

\author{
Michael D. Watson ${ }^{1}$, Amy Epps $^{2}$, and Van Woodruff ${ }^{3}$ \\ NASA Marshall Space Flight Center, Huntsville, AL 35812 \\ Michael Jacob Vachon ${ }^{4}$ \\ NASA Johnson Space Center, Houston, TX 77058 \\ Julio Monreal ${ }^{5}$ \\ European Space Agency, Launchers Directorate, Paris, France \\ Marl Levesque \\ United Launch Alliance, Vandenberg AFB \\ and \\ Randall Williams ${ }^{7}$ and Tom McLaughlin ${ }^{8}$ \\ Aerospace Corporation, El Segundo, CA 90245
}

\begin{abstract}
Launch vehicles within the international community vary greatly in their configuration and processing. Each launch site has a unique processing flow based on the specific launch vehicle configuration. Launch and flight operations are managed through a set of control centers associated with each launch site. Each launch site has a control center for launch operations; however flight operations support varies from being co-located with the launch site to being shared with the space vehicle control center. There is also a nuance of some having an engineering support center which may be co-located with either the launch or flight control center, or in a separate geographical location altogether. A survey of control center architectures is presented for various launch vehicles including the NASA Space Launch System (SLS), United Launch Alliance (ULA) Atlas V and Delta IV, and the European Space Agency (ESA) Ariane 5. Each of these control center architectures shares some similarities in basic structure while differences in functional distribution also exist. The driving functions which lead to these factors are considered and a model of control center architectures is proposed which supports these commonalities and variations.
\end{abstract}

\section{INTRODUCTION}

Launch vehicles in both Europe and the United States have been operating successfully for several decades. The current members of these launch vehicle families include the new NASA Space Launch System (SLS), Delta IV, Atlas V, and Ariane 5. The operational success of these launch vehicles is reflected in their operational functions

\footnotetext{
${ }^{1}$ System Engineer, Mission Operations Laboratory, NASA Marshall Space Flight Center

${ }^{2}$ SLS Engineering Support Center Lead, Mission Operations Laboratory, NASA Marshall Space Flight Center

${ }^{3}$ SLS Ascent Operations Analysis Lead, Mission Operations Laboratory, NASA Marshall Space Flight Center

${ }^{4}$ Mission Operations Directorate, NASA Johnson Space Center

${ }^{5}$ ESA's Ariane 5 Operational Launch System Manager and former Launch Range Operations Manager at CSG.

${ }^{6}$ Launch Conductor, Launch Operation Division, United Launch Alliance

${ }^{7}$ Systems Director, Civil and Commercial Launch Projects, The Aerospace Corporation

${ }^{8}$ Project Lead, Space Launch Operation, The Aerospace Corporation
} 
and control center architectures. Each of these vehicles is surveyed below followed by an analysis of their similarities and identification of the driving characteristics in operational function distribution among the various control centers.

\section{Descriptions of Control Centers Architectures}

\section{A. NASA Space Launch System (SLS)}

The NASA SLS control center model includes the Launch Control Center (LCC) located at Kennedy Space Center in Florida, the Mission Control Center - Houston (MCC-H) at Johnson Space Center in Texas, and the SLS Engineering Support Center (SESC) located in the Huntsville Operations Support Center (HOSC) at Marshall Space Flight Center in Alabama. These three control centers operate cooperatively to support launch and ascent flight operations. Final vehicle integration, full integrated testing, and launch operations are led out of the LCC with engineering support for the SLS from the SESC. Flight operations are led out of the MCC-H with engineering support for the SLS out of the SESC. This provides a configuration where launch activities are controlled at the launch site, ascent flight is integrated with the Orion operations control located at JSC, and a common engineering support team supporting all SLS prelaunch, launch, and flight operations from the MSFC where the engineering force is located.

\section{Launch Control Center, Kennedy Space Center}

The Launch Control Center (LCC) at the Kennedy Space Center in Florida is a multi-vehicle launch site that supports the final assembly of major non-commercial flight vehicles, their fully integrated testing, and performs processing through launch. Pre-launch processing begins with integrated testing in the Vehicle Assembly Building (VAB) and continues through the movement of the vehicle on the Mobile Launcher (shown in Figure 1) to the Launch Pad.
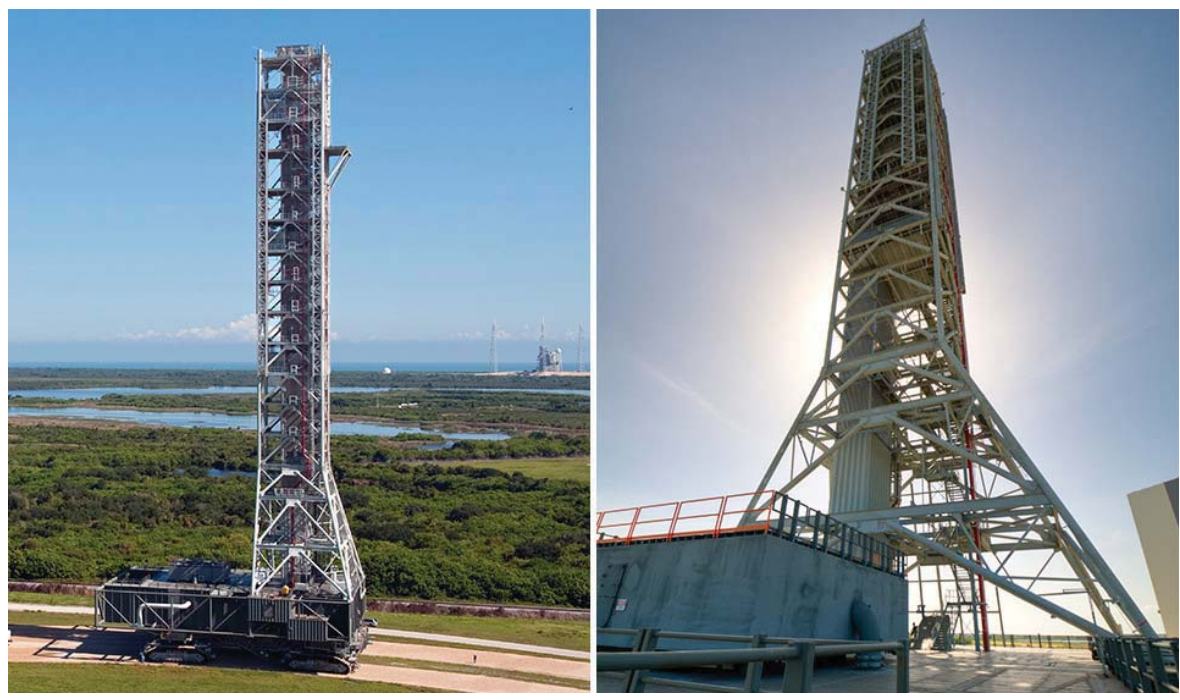

Figure 1: Newly Design Mobile Launcher for SLS (KSC)

SLS Launch Operations are conducted by the Ground Systems Development and Operations (GSDO) Operations Support Team from the KSC LCC. The SLS Launch Operations Support Team will be located in the HOSC SESC. During the launch countdown, SLS and the Multipurpose Crew Vehicle (MPCV) systems are powered up, configured to support launch, and monitored by the LCC, MCC-H, the SESC, with remote support from vehicle element (i.e., booster, engines, core stage, and integrated payload and spacecraft element) engineering support groups. The Launch Control System (LCS) monitors the flight vehicle (SLS and MPCV) and ground systems for launch commit criteria violations. Launch site weather is also monitored and weather briefings are provided by the weather officer to the Launch Director and the Mission Management Team. ${ }^{1}$

A GO/NO-GO launch decision is made based on evaluation of compliance with all launch commit criteria, flight rules, and Range Safety rules. Terminal countdown concludes with the lift-off of the launch vehicle and the 
separation of T- 0 umbilical lines and any other interfaces. It is at this point that the $\mathrm{MCC}-\mathrm{H}$ assumes control of the vehicle for ascent, and the SESC team becomes the engineering support for ascent to the MCC-H. ${ }^{2}$

\section{Mission Control Center, Johnson Space Center}

The Mission Control Center - Houston (MCC-H) at the Lyndon B. Johnson Space Center (JSC) supports the ascent flight operations for the SLS. Flight operations begin at launch and go through separation of the Orion from the SLS core stage and the splash down of the core stage down range. $3,4,5,6$

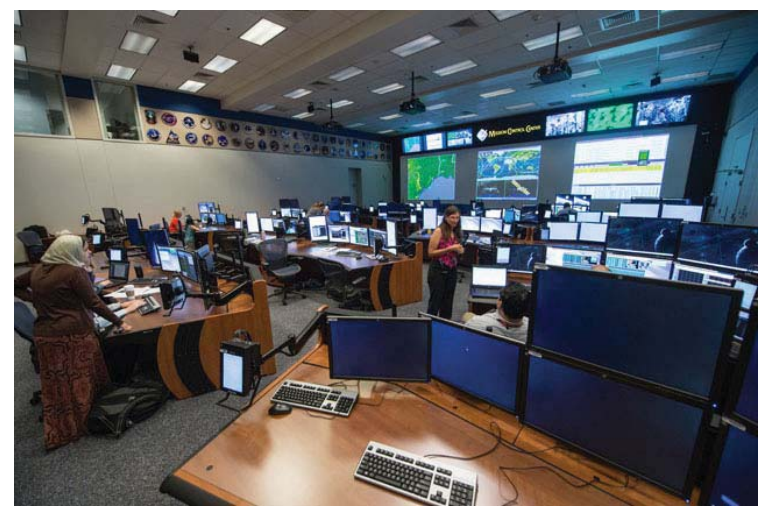

Figure 2: Mission Control Center, Houston Texas (MCCH) Exploration Control Center

The MCC-H is a multi-vehicle control center (Figures 2 and 3) and supports a broad array of spaceflight operations and mission classes including flagship programs; crewed and un-crewed technology demonstrations; robotic and human-robotic missions to Geocentric orbits, LaGrange Points, and lunar activities; expanded science utilization of the International Space Station (ISS); and commercial crew and cargo missions to the ISS. The MCC-H provides support of all mission phases of spaceflight operations, including pre-flight; (both pre-integration and post integration); ascent; orbit; orbital transfer; assembly; rendezvous, proximity operations, and docking (RPOD); entry, descent, and landing (EDL); and recovery.

In support of these spaceflight operations, the MCC-H operates under rigorous operations standards and processes that preserve the integrity of each vehicle design and manage

operational risks. These standards and processes assure the safety of the flight crew, the public, and the vehicle by ensuring mission planning, procedure execution, personnel training, and operation of the vehicle(s) are consistent with the end-to-end system design capabilities and limits. Effective real-time launch and flight operations assure the ability to make timely and informed decisions to continue or terminate a mission based on the full understanding of the immediate and future risks.

The MCC-H provides a set of functional capabilities for command, control, and support of launch vehicles and their spacecraft and/or payload. These capabilities are implemented through multiple subsystems which provide mission critical and mission support functions. The mission critical functions are spacecraft command and control

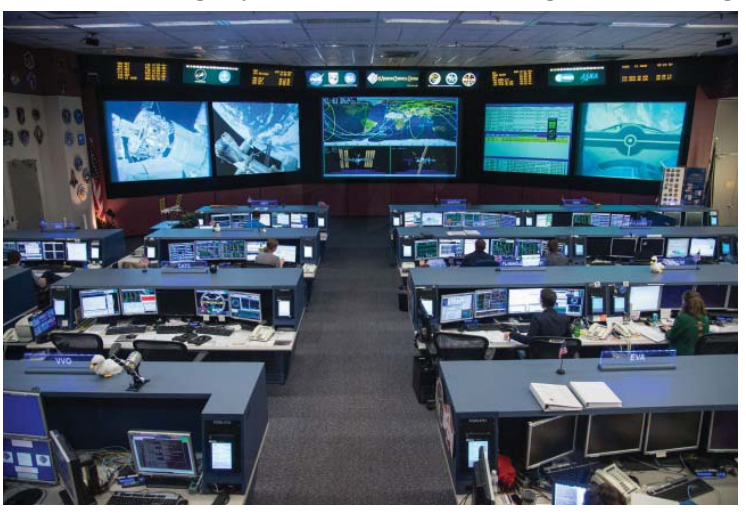

Figure 3: Mission Control Center, Houston, Texas International Space Station Control Center capabilities which include:

- Acquire telemetry downlink data from White Sands (via TDRSS), process, display and distribute the acquired telemetry downlink data

- Generate, send, and verify command uplink data

- Acquire, process and distribute trajectory, timing, voice, video and other ancillary data

- Record, store and distribute the above mission data

The mission support functions include integration, training and certification, product development and analysis capabilities which include:

- Coordinate external interfaces for real-time mission support

- Provide personnel training and certification standards

- Develop flight management and operations products and procedures

- Perform pre-mission planning and real-time mission re-planning

- Provide vehicle subsystem modeling for monitoring, evaluation, and analysis

- Provide facilities for development, integration, analysis and test of functionalities

- Provide anomaly tracking and resolution

- Enable remote access and utilization of the above capabilities 


\section{Flight Control Team}

Flight controllers are trained and certified to perform specific functions with clearly defined roles and responsibilities during spaceflight missions. Examples of these functions include command, control, and monitoring of the launch vehicle and spacecraft during ascent, orbit, and entry operations as well as unique operations tasks related to robotics manipulation, Extravehicular Activity (EVA), and rendezvous/docking. Individual flight controllers are assigned to vehicle subsystems or mission tasks based on their expertise and training. A group of flight controllers that covers all of the necessary functions to ensure mission safety and success are called a Flight Control Team (FCT). The FCT is trained as a cohesive unit that is maintained through completion of the spaceflight mission or mission phase.

The FCT is responsible for both the SLS and MPCV mission execution including overall execution of the mission and assurance of crew and vehicle safety, command and control of vehicle systems, mission re-planning, trajectory design and maintenance, robotics or EVA planning and execution, and maintenance and operations of the control center. The FCT normally consists of a Flight Director responsible for leading the FCT; vehicle system operators and experts (both SLS and MPCV); payload operators and experts; EVA, robotics, and crew systems experts; trajectory analysis, design, and operation experts (including ascent flight, orbital insertion, orbital maneuvers, and on-orbit debris avoidance); planners responsible for vehicle (SLS and MPCV)/crew activities, realtime re-planning, and integration of external stakeholders; a Flight Surgeon responsible for monitoring and evaluating the health of the onboard crew; a Capsule Communicator (CAPCOM) to act as a liaison between the onboard crew and the FCT; representatives from the Spaceflight Meteorology Group (SMG) that provide weather observation and forecasting support in support of launch and landing; a Ground Controller responsible for maintenance and operation of the MCC-H and support facilities; and a Public Affairs Officer.

Each spaceflight mission will have a unique set of mission objectives, supporting subsystems, and vehicle elements. Vehicle system operators and experts are provided based upon an assessment of the overall mission and the vehicle(s) that will be used to support the mission. Pre-flight training will be tailored to prepare the FCT to execute mission rules and respond to failures that may be unique to a particular mission, subsystem, or vehicle. For example, to fly missions with the MPCV spacecraft, the FCT will be initially structured to provide launch vehicle insight appropriate for the first test flight of MPCV on the un-crewed EFT-1 mission, which utilizes a Delta IV Heavy vehicle with limited command and control capabilities,. The FCT will be restructured to provide greater insight and vehicle command and control functions for subsequent crewed flights of MPCV on the SLS, which will have additional capabilities and FCT responsibilities.

Almost all FCTs are organized into 'front room' and 'back room' support personnel which provide support for the SLS and MPCV vehicles. Front room flight controllers support consoles in the primary Flight Control Room (FCR) and are responsible for mission integration and monitoring the vehicle(s) at a system-level. Back room flight controllers work in Multi-Purpose Support Rooms (MPSRs), locally or remotely, and are generally responsible for monitoring the vehicle(s) at a sub-system-level. Additional teams provide flight support to the FCT, as required, but do not require the same level of training and certification as FCT personnel. These teams provide engineering support for specific vehicles or vehicle subsystems from within the MCC-H or from external sites; operate specific science or payloads; operate MCC-H facilities; and provide an interface to external organizations such as other NASA Centers, International Partner FCTs, the US government, and Private Industry. Ops Suites are also available and are designed to accommodate customers whom require integration with the rest of MCC-H but also require a standalone control room that can be isolated to protect proprietary information.

Integration with other Control Centers

The Flight Control Team for a particular mission may include flight controllers at one or more other control centers. The MCC-H maintains real-time interfaces with other control centers as required on a per-mission basis, or in the event of an emergency. These interfaces may include other NASA Centers, International Partners, US Government Agencies, and Private Industry. Each external control team is individually organized, based on organizational considerations internal to that center's program needs and heritage.

\section{NASA Launch Control Center (LCC)}

In support of launch operations, the LCC coordinates Eastern Range assets, including forward and return links within line-of-site of the vehicle, tracking, and search and recovery forces. The MCC-H coordinates broader global and in-space network assets, spaceflight meteorology, and Day-of-Launch I-Load files.

During the pre-launch timeframe, when the launch vehicle and spacecraft are being powered up and fueled for flight, the MCC-H and LCC maintain critical real-time interfaces to ensure the flight hardware, mission support personnel and services, network assets, spaceflight meteorology, range, and search and rescue forces are all well- 
coordinated. These interfaces include flight vehicle telemetry, countdown clock/Mission Elapsed Time, file transfer capability for weather data and vehicle initialization loads, launch vehicle navigation data, tracking data, voice, video, and supporting systems data.

\section{Alternate Control Center}

The Alternate Control Center (ACC) provides backup, temporary, and emergency control center capabilities. When the ACC is activated, the MCC-H operations instance hosted at JSC will transfer to the ACC located in the HOSC at MSFC. Depending upon the specific scenario being discussed, operators will perform mission operations and support activities (e.g., their console duties) from the HOSC/ACC itself, from JSC (either from the office environment or from consoles in the MCC-H), or from locations remote to both JSC and the HOSC/ACC. These scenarios will be possible because the HOSC/ACC will be provided with the same remote access and security capabilities deployed within the MCC-H.

International Partner Launch Vehicle Control Centers

Depending upon the mission, the MCC-H may support real-time connectivity with several International Partners (IP) including voice, video, and data. The Flight Control Team for a particular mission may include flight controllers at several IP control centers. Each IP control team is individually organized, based on organizational considerations internal to that IP's program needs and heritage.

\section{European Space Agency (ESA) - Automated Transfer Vehicle (ATV-CC)}

The ESA has their ATV Flight Control Team (ATV-CC) located at the French Space Agency (CNES) in Toulouse, France. This control center may participate as part of the integrated FCT for Exploration Missions and would provide augmented vehicle specialist insight into the MPCV Service Module (SM), which is being built by ESA based upon the ATV design. The engineering support team for this module would be located in France and report to MCC-H throughout the MPCV flight. MCC-H will serve as a bridge for data from MPCV SM to the ATVCC.

Commercial Cargo and Crew Partner Launch Vehicle Control Centers

For commercial flights to the ISS, the MCC-H supports real-time connectivity with the mission control centers of several Commercial Partners, which includes voice, video, and data. Under current contracts, these commercial flight control teams are located at MCC-H, MCC-D in Dulles, VA, and MCC-X in Hawthorne, CA. Similar to the NASA model, these partners maintain separate launch control centers at Wallops Flight Facility, VA and Cape Canaveral, FL.

For these flights, MCC-H has primary responsibility for overall mission integration, which can include routing of critical pre-flight mission information such as mass properties, mission rules, and cargo manifest to the Russian partner as required for ISS command and control. MCC-H also ensures that Commercial flights do not conflict with planned activities of all International Partners. MCC-H direct support to the Commercial Partners includes helping accomplish and plan real-time execution of spacecraft trajectory operations, scheduling TDRSS time, and real-time evaluation of failures. MCC-H has also served as a bridge for data from MCC-X to the Payload Operations Integration Center (POIC) located in the HOSC in Huntsville, AL.

\section{3. $\quad$ SLS Engineering Support Center (SESC), Marshall Space Flight Center}

The Space Launch System (SLS) Engineering Support Center (SESC) is located within the Huntsville Operations Support Center (HOSC) building at the Marshall Space Flight Center (MSFC) in Huntsville, Alabama shown in Figure 4. The SESC is the next generation engineering support facility for the Space Launch System (SLS). The purpose of the SESC is twofold: and

(1) Provide pre-launch operations support to the Launch Operations Team at the Kennedy Space Center (KSC);

(2) Provide flight operations engineering support to the Mission Control Center (MCC) at the Johnson Space Center (JSC). The SLS Engineering Support team will provide a consolidated team supporting operations from start of countdown through disposal of SLS Elements. 


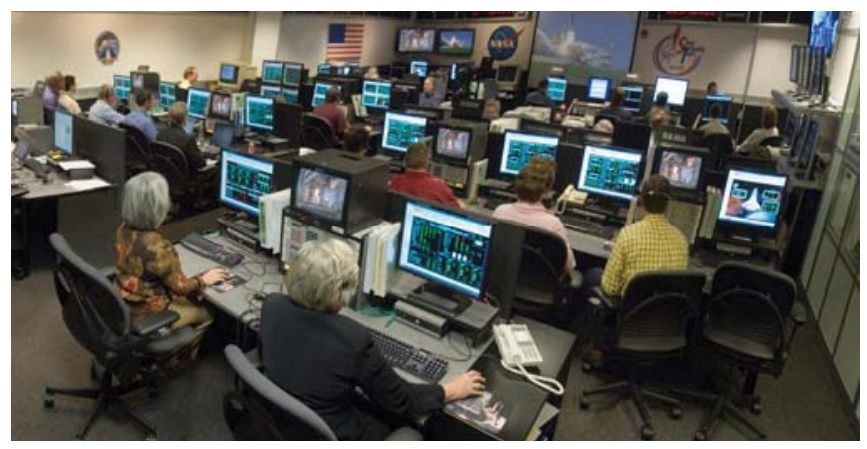

Figure 4: Space Launch System (SLS) Engineering Support Center (SESC)

SLS launch operations will be conducted by the Ground System Development Office (GSDO) Launch Operations Team, which will originate from the Kennedy Space Center (KSC) Launch Control Center (LCC). The SLS Engineering Support Team, located in the HOSC SESC, will provide overall launch support to the GSDO Launch Operations Team. The SLS Engineering Support Team consists of the responsible system engineers, program managers, chief engineers, safety engineers, and SLS Element subject matter experts. SLS Engineering Support Team members will assess the SLS vehicle state and provide engineering support as required by GSDO Launch Operations Team. Remote services will be provided to bring up SLS engineering discipline and vehicle element experts from manufacturing or engineering sites for specific pre-launch/launch and ascent flight activities as needed. These remote services will include SLS Element engineering sites located at contractor facilities as appropriate. SLS personnel will support day of launch operations as part of the Launch Authority Team (LAT). The LAT will be the authoritative source for committing the SLS vehicle for launch. ${ }^{1,7}$ Specific responsibilities of the SLS Engineering Support Team include:

- Monitor SLS vehicle countdown activities

- Respond to and assist in launch commit criteria violation resolutions with the LCC as required

- Provide SLS vehicle status to SLS Program Manager and LCC as required

- Participate in Launch Go/No-Go polling with LCC and MCC.

- Provide membership to the Launch Authority Team (LAT)

- Provide SLS input into launch scrub turnaround activities

Ascent and on orbit flight operations are conducted from the MCC-H. The SLS Engineering Support Team will transition to the SLS Flight Operations Support Team after liftoff and support the mission through ascent and disposal of the SLS vehicle elements. The SLS Flight Operations Support Team will perform the Mission Evaluation Room (MER) function and serve as the backroom support team to the MCC-H. ${ }^{7}$ Specific responsibilities of the SLS Flight Operations Support team include:

- Provide SLS vehicle support to the MCC-H

- Monitor the SLS vehicle during ascent and on orbit operations

- Provide SLS vehicle status during ascent and on orbit operations to the MCC-H as required

- Provide SLS vehicle Flight Rule coordination with MCC-H as required

- Participate in SLS in-flight vehicle anomaly analysis with the MCC-H as required

- Participate in post flight SLS vehicle analysis with the MCC-H as required.

Figure 5 shows the SLS day of launch operations concept with the responsibilities and functional interfaces between the various NASA control centers.

The SLS Flight Operations Support Team and SLS Engineering Support Team will be certified for console operations and will be provided with

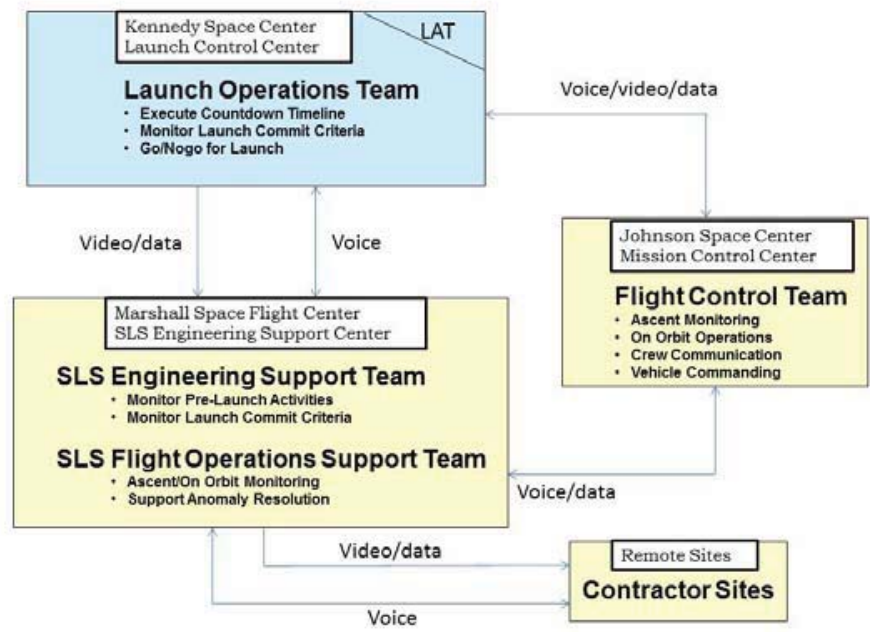

Figure 5: Space Launch System (SLS) Day of Launch Operations Concept console positions including voice, imagery, data, and operations tools necessary for launch and flight support. Sustaining engineering expertise will be provided with remote service including voice, imagery, and data. The sustaining engineering expertise will be trained on the remote services and will not be certified for launch operations. Training positions will be supported for the Flight 
Operations Support Team, and the SLS Engineering Support Team will be provided console seats. Observers will be provided access to the SESC only with approval of the SESC Operations Manager.

The SESC will have the capability to provide engineering services for a variety of activities in addition to launch and flight operations. These include remote support to system testing, vehicle testing, training, and simulation. SESC will also have the capability to provide extended operational support for problem resolution resulting in launch scrubs. The SESC will have the capability to reduce and archive all of the SLS flight and test telemetry and provide the services for stakeholders to retrieve this data for post flight analysis.

\section{B. United Launch Alliance (ULA) DELTA IV and Atlas V Launch Systems}

Launch Operations for the Atlas V and Delta IV programs by United launch Alliance (ULA), the joint venture LLC formed in 2006 from the heritage Evolved Expendable Launch Vehicle (EELV) programs of parent companies Boeing and Lockheed Martin, conduct launch operations from Cape Canaveral Air Force Station (CCAFS) Florida and Vandenberg Air Force Base California (VAFB). The design center in Centennial Colorado also provides launch day support for both programs. At CCAFS, Launch Complex 41 (LC41) and Launch Complex 37 (LC 37) support the Atlas V and Delta IV programs, respectively. Similarly at VAFB, the launch complexes are Space Launch Complex 3 East (SLC 3E)-Atlas V and Space Launch Complex 6 (SLC 6) -Delta IV. Additionally VAFB houses the Delta II Launch Complex SLC 2 West.

At CCAFS, the Launch Control Centers (LCC) are located in the Atlas Space Operations Center (ASOC) and the Delta Operations Center (DOC). The LCCs are independent locations generally dedicated to each program. They both house engineering, support and management office spaces, the LCCs and launch vehicle off-stand processing areas. The ASOC is capable of storing multiple launch vehicles simultaneously as well as conducting limited horizontal testing of one launch vehicle. The DOC similarly accommodates non-powered testing of the Delta IV upper stage in a vertical orientation. At LC 37, a Horizontal Integration Facility (HIF) allows processing and mating of the Delta IV Common Booster Cores (CBC). At VAFB a similar HIF supports the functions of the CCAFS HIF as well as the launch vehicle testing role of the DOC.

In addition to the above noted functions, both the ASOC and DOC contain an Engineering Support Area and a Mission Directors Center (MDC). The Engineering Support Area provides day of launch data and communications access to design center engineers who participate final close out inspections at the launch sites. The MDC provides data and communications access to participating organizational managers from launch vehicle, spacecraft and government entities who participate in final readiness reviews at the launch base. A Mission Integration Team (MIT) room also provides data and communications access for spacecraft and launch vehicle mission engineering to coordinate day of launch integration issues.

At VAFB, the three LCCs are co-located in a common Remote Launch Control Center (RLCC). The LCCs themselves occupy different control rooms within the facility however the MDC, MIT and to a limited extent the Engineering Support Area support all programs. This is facilitated by the use of a common Atlas heritage data display system the Automated Data Monitoring System (ADMS). ADMS is available in all ULA facilities enabling participation in data monitor of any operation from any location. ADMS forms the infrastructure for the Atlas launch control system whereas Delta IV launch control consists of the Delta Launch Processing system (DLPS) and the Delta II launch control consists of the Computerized Data Processing System (CDPS). Each launch control center provides the operations engineering staff and management the capability for Ground Command, Control and Communications (GC3) operations. GC3 provides interface between propellant transfer and avionics control operators in the LCCs and remote terminal equipment at each launch complex. Additional support systems include voice, video and timing provisions and launch base range interfaces with 45th Space Wing (CCAFS) and 30th Space Wing (VAFB) elements.

At CCAFS, the NRO/USAF maintains a Technical Support Facility (TSF) and NASA Launch Services Program maintains Hanger AE for government launch vehicle technical support operations separate from the ULA facilities. These facilities have the capability to receive and display launch vehicle data for government engineering evaluation. VAFB RLCC contains a similar function as the TSF known as the Launch Vehicle Launch Support Center (LVLSC). This location supports all ULA launch operations at VAFB as well as providing the capability to "shadow" operations from CCAFS launch sites for training purposes. Similar to Hanger AE, NASA operates Building 836 at VAFB.

For Launch day operations, the ULA Denver facility contains a Denver Operations Support Center (DOSC) is shared by both the Atlas and Delta programs. This facility provides similar capabilities as the Engineering Support Area for design center engineers who are not required to travel to the launch sites. 
Spacecraft control operations typically are autonomous from the launch vehicle day of launch LCC operations. However, provisions exist in the DOC, ASOC and RLCC for spacecraft ground support operations. In all locations, the MIT rooms accommodate direct coordination of launch vehicle and spacecraft integration engineering functions during countdown operations. Spacecraft remote operations electrical interfaces are provided by ULA at each launch complex from the connection of broad area infrastructure at either CCAFS or VAFB. This is accommodated by Payload Users Rooms (PLUR) at each VAFB launch site and LC 37 or the Payload Van (PVan) LC 41. The mobile nature of operations at LC 41 dictates the use of this transportable equipment interface. Spacecraft typically emplace their remote monitoring equipment at a processing facility of a government or commercial entity such as the NRO, NASA or Astrotech Space Operations at which spacecraft initial preparations are performed following delivery from the spacecraft contractor's factory and prior to integration with the launch vehicle. This equipment used for initial spacecraft operations is often then utilized for on pad operations. Spacecraft supplied remote equipment is installed in the PLUR or PVan and connected to the launch vehicle umbilicals via ULA provided termination points. Day of Launch spacecraft operations are conducted separately but in parallel with launch vehicle operations but procedurally coordinated for critical functions such as time of launch coordination, internal power transfer, final configuration for launch and countdown hold/abort function.

The facilities described herein apply to current unmanned operations. Aspects of ULA operations support for human spaceflight are expected to incorporate a significant amount of this infrastructure in addition to aspects yet to be emplaced for unique manned mission requirements. ${ }^{8}$

\section{European Space Agency (ESA) Ariane 5}

After qualification of the Ariane 5 launch system (i.e. launch vehicle system and its launch complex), the European Space Agency entrusted Arianespace for carrying out the utilization phase of the launch system. ESA has an agreement with the French National Space Studies Centre (CNES) to use and maintain the launch range site in French Guiana for ESA's launchers operations. Indeed Ariane 5 is launched from the Guiana Space Center (CSG) in French Guiana, Europe's spaceport. CSG includes the launch range, spacecraft preparation and launch complex facilities, commodities and services required to assemble, integrate, checkout, launch, track and monitor the Ariane 5 , Vega and Soyuz launch vehicles and their payloads.

Two main control centers are involved in an Ariane 5 launch: the Launch Vehicle Preparation Control Center, "Centre de Lancement" in French (CDL) and the Launch Mission Control Center (LMCC) called Jupiter-2 from the name given to the building enclosing the LMCC. Additional control rooms located in the payload preparation facilities (EPCU) are used by the customer teams for preparing the spacecraft and their own net-work for launch.

The CDL3 contains the "blockhouse" (Figure 7) and acts as the launcher preparation control center for Ariane 5 launchers. An Arianespace launch operations team lead by the Launch Complex Operations Manager (COEL) is responsible for all launcher related operations. An engineering team lead by Arianespace's Ariane Production Project Manager (CPAP) and including personnel from main industrialists (i.e. Airbus, Snecma, Europropulsion), sit in a separate room within the CDL3 to monitor launch vehicle systems including the cognizant subsystem engineers, who are responsible for providing a first assessment and way forward in case of trouble during the standard operations. Remote access is available for engineers at the manufacturing/design sites to support troubleshooting if necessary. These engineers have the same screens as in the CDL3 to see the data in the same format. Thus, engineering primary support is provided at the launch control center, with more detailed engineering support from the manufacturing and engineering sites in Europe in an "off line" support capacity. These remote engineers are called in as needed to support troubleshooting independent of the launch operations countdown. The COEL provides the vehicle Go/No Go to the Launch Range Manager (DDO) siting in the LMCC $12 \mathrm{~km}$ away from the CDL.

The Launch Mission Control Centre Jupiter-2 (Figure 8), is first the launch range operations control center and acts as well as overall coordination center for all activities converging to the launch readiness: launcher, spacecraft and range operations for launch. So, in addition to the DDO and its launch range team, the Spacecraft Customer Managers and Arianespace's Launch Mission Director (CM) are sitting close to the DDO in Jupiter-2. 

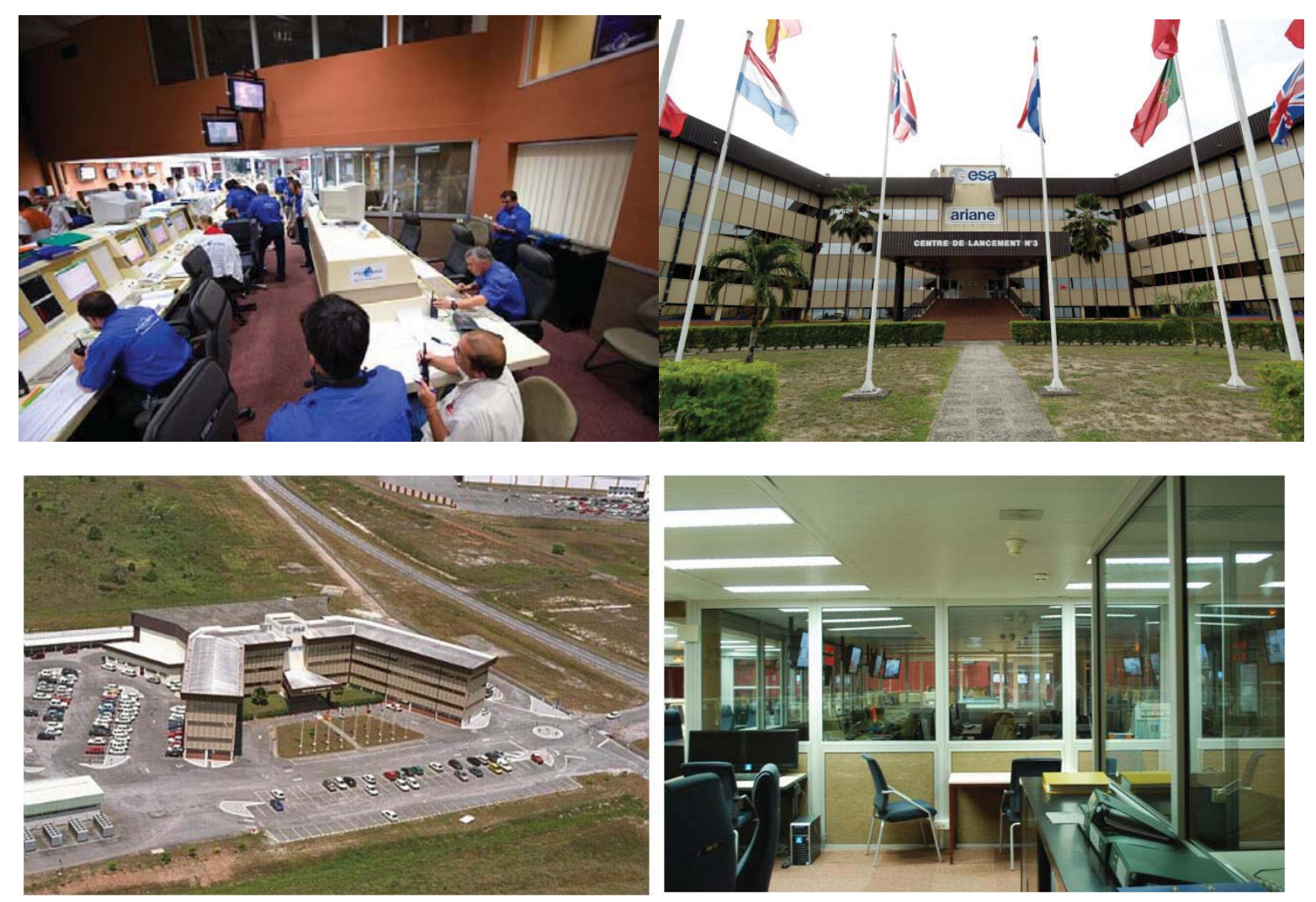

Figure 6: CDL3 and View inside Blockhouse

Readiness for launch is given to the DDO and to the CM by the COEL concerning the launcher and to the CM by the spacecraft customer's representatives concerning the spacecraft. The CM transfers Arianespace's Management launch decision to the DDO. Authorization to launch is eventually provided by the CNES high level Safety Authority (i.e. CSG Director) to the DDO who lets the launcher final automatic sequence proceed (the so called Synchronized Sequence) until liftoff.

Logistics, weather forecast, real-time communications, telemetry acquisition and trajectory tracking, for launch preparation, liftoff, ascent flight and in-orbit maneuvers, are provided by the launch range team coordinated by the DDO from Jupiter-2. Several ground stations around the globe (i.e. Brazil, Ascension islands, Gabon, Kenya or other specific stations for non-GTO launches) are coordinated via dedicated communication links with CSG in Kourou and in particular with Jupiter-2 controllers.
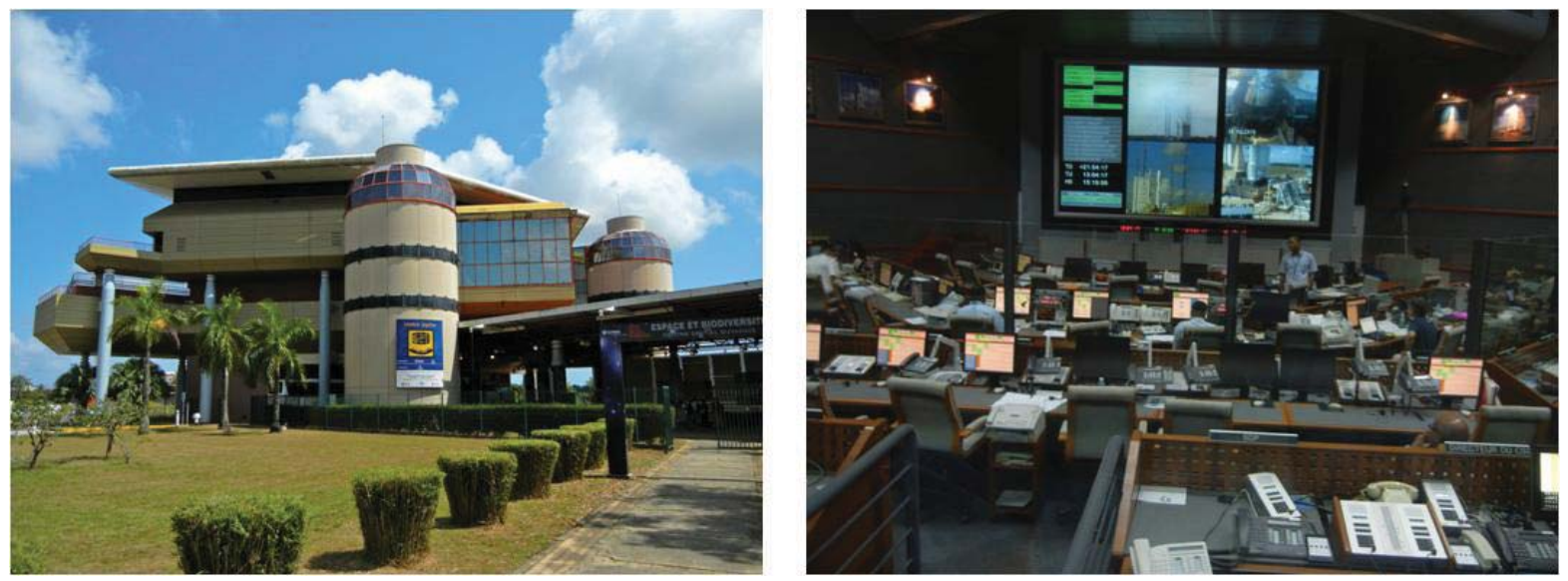

Figure 7: Launch Mission Control Center, Jupiter-2 Building

Following separation from the Ariane 5, payload in-orbit operations are conducted from a separate facility provided by the payload owner. 
During the ascent flight and until separation of the satellites, a real time quick-look check of vital parameters is performed by an Arianespace lead small engineering team. The day after the launch, main parameters are assessed at CSG by this engineering team which compares recorded data to specifications for major ones. After this preliminary post-flight analysis, the complete data records are provided by CNES to Arianespace for a more detailed post flight assessment performed by all concerned industrialists in Europe. Actions are taken within the week following the launch to clear any anomaly that could impact the following launches.

\section{Commonalities and Differences between Center Models}

Examining each of the control center interactions for the SLS, Delta IV, Atlas V, and Ariane 5 a common framework emerges with three operational functions mapped into multiple control centers with differences in operational details. Each of the different launch vehicles has basically a three operational control functions: launch control, engineering support, and payload control. These functions are supported by a multiple control center model which provides for separation of each of the operational functions within the context of geographical constraints, crewed missions, shared flight control teams, and mission life times.

For un-crewed payload missions prelaunch, launch, and flight operations are controlled from a single control center. This is located at the launch site allowing the engineers in the control center to support assembly and prelaunch operations. Geographically, the design engineers are not located at the launch site. These are typically at a manufacturing site or a centralized corporate engineering location. Thus, the Engineering Support Center is located with the engineering work force. This cuts down significantly on travel and provides ready access to any detailed engineer to work specific problems as part of prelaunch preparations. There can be multiple Payload Control Centers depending on the number of payloads carried on a given launch. Payload Control Centers are located at various corporate locations established for various reasons depending on the payload(s). These control centers are active for the entire duration of the mission, starting with prelaunch activities through end of the payload mission. Payload operators have various corporate reasons for the location of a Payload Control Center including skilled personnel availability, personnel support services in the local communities, and access to reliable communications assets. The separate launch vehicle and payload control centers allows the launch vehicle control team to be flexible in supporting a variety of different payloads and to drop off quickly (once payload separation occurs) and begin processing the next vehicle. This provides a very efficient model for launch vehicle operations.

SLS has the same three operational function philosophy, but divides the functions slightly differently. All prelaunch and launch operations are managed by the LCC located at the launch site. The MCC-H provides flight operations support to the LCC during these operations and the SESC provides the SLS engineering support. At lift off, operational responsibility transfers to the MCC-H with engineering support continuing from the SESC. For human spaceflight, this provides an integrated Flight Control Team (FCT) for the SLS and the MPCV during ascent flight. This integrated and co-located FCT provides an enhancement to flight safety as decisions for flight are made by an integrated team taking in consideration both crew impacts and integrated vehicle stack (i.e., SLS and MPCV) constraints. For cargo-only missions, this configuration is also utilized as the FCT is already assembled and trained to support the launch vehicle ascent operations. A smaller FCT will be used for cargo missions, as the MPCV/crew support functions of the FCT will not be required. For these cargo missions, a separate payload control center will also be involved which may be at the MCC-H, HOSC, or other customer-based facilities depending on the specific payload. Depending on the mission, multiple Payload Control Centers may be supported.

ULA has a common operations approach for launching the Atlas V and Delta IV. Launch and flight control are located at the ASOC (Atlas V) or DSOC (Delta IV) at the launch site. A small engineering support team is located in the ASOC or DSOC. Similarly, at the VAFB site, the RLCC hosts the launch control functions. Additional engineering support is located at the DSOC in Denver for both launch sites and provides engineering support throughout the launch and flight operations. This provides a single Engineering Support Center supporting multiple launch sites. This configuration significantly cuts down on travel of the engineering force and time lost in either supporting a mission when not needed or in holding a mission to obtain the necessary engineering support. The payload operator(s) have separate payload control center(s). These payload control centers are designed for longterm (several years) operations and are located geographically based on corporate priorities in the operations of their specific payload.

Ariane 5 also supports a multiple control center approach with a different split in responsibilities. Launch vehicle preparation and readiness for launch operations are conducted from CDL3 launcher preparation center at the launch complex site (ELA3). A small engineering support team sits in the CDL3, with access to a larger support team in an off line fashion at the manufacturing and engineering sites in Europe. Payload preparation for launch is performed $12 \mathrm{~km}$ away from the CDL3 in dedicated customer control rooms at CSG in the EPCU buildings. Launch range own 
preparation for launch and overall launch readiness coordination is done from Jupiter-2 Launch Mission Control Center also located $12 \mathrm{~km}$ away from the CDL3 at the launch range site. Launch decision and authorization managers for the launcher, spacecraft and launch range are sitting at the LMCC. Once the payloads have separated from the launch vehicle, then separate payload control facilities take over the in-orbit operational control of each payload.

There are several factors contributing to the distribution of the operational functions across multiple control centers. Geographical location is a key factor. Launch control centers are located at the launch site for access to the vehicle and payloads during assembly and launch. Engineering support is located at manufacturing sites or design centers to minimize the travel and work disruption of the vehicle manufacturing and design engineering work force. When necessary, a small group may travel, often with remote support at the manufacturing and design center location(s). Geographic considerations also play into Payload Control Center locations which are driven by access to skilled personnel, local community support, and reliable communications. Mission duration is also a factor. Launch vehicle operations are primarily preflight and mission flight times are only a few minutes. However, payload mission durations span years. Thus it is more advantageous to have separate control centers, one focused on launch site activities and one focused on long duration mission activities. This frees the launch site to continue support for the next mission once the vehicle has cleared the pad and the payload control center to focus on the very different pace of long term mission operations. Crewed missions bring in another factor where integrated flight operations of the launch vehicle and crew module enhance crew safety through integrated decision making across the entire vehicle stack (launch vehicle and crew capsule). This is an important factor to consider for future crewed vehicle missions.

\section{Summary}

Common control center operational functions exist among the various launch vehicles considered. These functions consist of launch operations, engineering support, and payload operations. The split of operations between multiple control centers varies in details slightly based on several factors. Launch control centers are located at the launch sites where the launch equipment is located. This provides an integrated launch operations and launch equipment support team at a single location. Geographical considerations play a large factor in the location of launch vehicle engineering support. These teams are typically located at the engineering facilities for the launch vehicle whether commercial or government. Human rated operations also plays a role where crewed vehicle and launch vehicle operations are combined to provide and integrated FCT working both vehicle and crew constraints during ascent flight. Mission duration also plays a role. Payload operations centers, similar to engineering support, are located in central locations where, for the long term (years), they can be located with access to skilled personnel, good community support, and reliable communications infrastructure. Each of these factors tend to lead to a set of three control centers for any launch vehicle mission.

\section{ACKNOWLEDGEMENTS}

The authors would like to thank Herbert D. Rice, LSX10, Kennedy Space Center for his many years of service to NASA KSC and his review of the launch site references.

\section{REFERENCES}

${ }^{1}$ GSDO-ACO-1010 Ground Systems Development and Operations Program Architectures and Concept of Operations Document, Revision B, Release Date: 4-29-2013

2 C3R-3008 Command, Control, Communications and Range Integrated Product Team Concept of Operations, Baseline Release Date: 6-24-2013.

${ }^{3}$ CxP 72134, Constellation Program Mission Operations Project Mission Systems Architecture Description

Document Rev A., August 12, 2009.

${ }^{4}$ CxP 72203, Constellation Program Mission Operations Project Mission Operations Architecture Description

Document Mission Class: Orion-to-ISS, June 2, 2010.

${ }^{5}$ JSC-36496-GEN, Final, MOD International Liaison Console Handbook RIO Operations, September 12, 2012.

${ }^{6}$ JSC-35067, Mission Control Center Systems (MCCS) Operations Concepts, December 07, 2010. 
${ }^{7}$ SLS-PLAN-020, Revision B Space Launch System Program Concept of Operations, Effective Date 4-12-2013. ${ }^{8}$ Laube, J.; Williams, R.; Ganguli, U.; Wyse, J.; McLaughlin, T.; Bucher, D.; Smith, S.; Yee, L.; McCall, S.; Brekke, J.; Ringler, S.; Taylor, T.; "Assessment of Launch Operations Architectures for the Space Launch System Program Office at Marshall Space Flight Center”, Aerospace Technical Report 2013(5738), 30 September 2012. 